\title{
Lagrangians for invariant sub-spaces of the squared Pauli-Lubanski vector
}

\author{
G. $\mathrm{Cabral}^{1}$, and M. Kirchbach ${ }^{2}$ \\ ${ }^{1}$ Facultad de Fisíca, Univ. Aut. de Zacatecas, A.P. C-600, Zacatecas, ZAC-98062, México \\ ${ }^{2}$ Instituto de Física, Univ. Aut. de San Luis Potosí, San Luis Potosí, S.L.P. 78240, México
}

\begin{abstract}
We present an alternative formalism to the Rarita-Schwinger framework for the description of "has-been" higher-spins at rest that avoids the Velo-Zwanziger problem.
\end{abstract}

Introduction. One of the long standing problems in particle physics is the covariant description of higher spin states. The standard formalism is based upon totally symmetric Lorentz invariant tensors of rank-K with Dirac spinor components, $\psi_{\mu_{1} \ldots \mu_{K}}$, which satisfy the Dirac equation for each space-time index. In addition, one requires $\partial^{\mu_{1}} \psi_{\mu_{1} \ldots \mu_{K}}=0$, and $\gamma^{\mu_{1}} \psi_{\mu_{1} \ldots \mu_{K}}=0$. The solution obtained this way (so called RaritaSchwinger framework) describes a "has-been" spin- $\left(K+\frac{1}{2}\right)$ particle at rest that is a parity singlet, i.e. without a companion of opposite parity, and a particle of indefinite spin in flight. Problems occur when $\psi_{\mu_{1} \ldots \mu_{K}}$ constrained this way are placed within an electromagnetic field. In this case, the energy of the "has-been" spin- $\left(K+\frac{1}{2}\right)$ state becomes imaginary, and it propagates acausally (Velo-Zwanziger problem [1]). Here we first emphasize that the $\gamma^{\mu_{1}} \psi_{\mu_{1} \ldots \mu_{K}}=0$ constraint is a short-hand of the definition of the above parity singlet as one of the invariant subspaces of the squared Pauli-Lubanski vector, $W^{2}$. We consider the simplest case of $K=1$ and construct the covariant projector onto that very state as $-\frac{1}{3}\left(\frac{1}{m^{2}} W^{2}+\frac{3}{4}\right)$. We suggest to work in the sixteen dimensional vector space, $\Psi$, of the direct product of the four-vector, $A_{\mu}$, with the Dirac spinor, $\psi$, i.e. $\Psi=A \otimes \psi$, rather than keeping space-time and spinor indices separated (as in $\psi_{\mu}$ ) and to consider $\left(-\frac{1}{3}\left(\frac{1}{m^{2}} W^{2}+\frac{3}{4}\right)-1\right) \Psi=0$ as the principal wave equation without invoking any further supplementary conditions. In gauging the equation minimally, calculating and nullifying its determinant, we obtained the energy-momentum dispersion relation. The latter turned out to be well behaved and free of pathologies, thus avoiding the classical Velo-Zwanziger problem.

Projectors onto $W^{2}$ invariant sub-spaces. The Pauli-Lubanski vector is defined as

$$
W_{\mu}=-\frac{1}{2} \varepsilon_{\mu v \rho \tau} S^{\nu \rho} P^{\tau},
$$

where $\varepsilon_{0123}=1$, and $P^{\tau}$ are the generators of translations. Its squared is calculated to be

$$
W^{2}=-\frac{1}{2} S \cdot S P^{2}+G \cdot G, \quad G^{v}=S^{\sigma v} P_{\sigma} .
$$


In $(s, 0) /(0, s), G^{2}=-W^{2}$ and $W^{2}$ reduces to $-m^{2} \mathbf{S}^{2}$. For fields of the type $\left(s_{1}, s_{2}\right)$ with $s_{1}$ and $s_{2}$ non-vanishing, $G^{2} \neq W^{2}$ and spin is no longer a good quantum label for such particles in flight. To build $W_{\mu}$ in $A_{\nu} \otimes \psi$ we recall generators of the Dirac representation

$$
S_{v \rho}^{\left(\frac{1}{2}, 0\right) \oplus\left(0, \frac{1}{2}\right)}=\frac{1}{2} \sigma_{v \rho}, \quad \sigma_{v \rho}=\frac{i}{2}\left[\gamma_{v}, \gamma_{\rho}\right]
$$

where $\gamma_{\mu}$ are the Dirac matrices. The Lorentz generators in the four-vector $\left(\frac{1}{2}, \frac{1}{2}\right)$ space are obtained from those of the right-handed $\left(\frac{1}{2}, 0\right)$, and left-handed $\left(0, \frac{1}{2}\right)$ spinors [2-4] in noticing that $\left(\frac{1}{2}, \frac{1}{2}\right)$ is the direct product of $\left(\frac{1}{2}, 0\right)$ and $\left(0, \frac{1}{2}\right)$. One finds [4]

$$
S_{0 l}^{\left(\frac{1}{2} \frac{1}{2}\right)}=1_{2} \otimes\left(i \sigma_{l}\right)+\left(-i \sigma_{l}\right) \otimes 1_{2}, \quad S_{i j}^{\left(\frac{1}{2} \frac{1}{2}\right)}=i \varepsilon_{i j k}\left(1_{2} \otimes \sigma_{k}+\sigma_{k} \otimes 1_{2}\right),
$$

where $\sigma_{k}$ are the Pauli matrices. Now the generators in $\psi_{\mu} / \Psi$ are cast into the form $[3,4]$ :

$$
S_{v \rho}=S_{v \rho}^{\left(\frac{1}{2} \frac{1}{2}\right)} \otimes 1_{4}+1_{4} \otimes \frac{1}{2} \sigma_{v \rho}
$$

In the finite dimensional representation space, $\left(\frac{1}{2}, \frac{1}{2}\right)$, where one replaces the Poincaré generators of translations, $P_{\mu}$, with their eigenvalues, $p_{\mu}$, i.e. by numbers, $\left[W^{\left(\frac{1}{2} \frac{1}{2}\right)}\right]^{2}$ is no longer proportional to the unit matrix and does not covariantly commute with the operator of the squared spin, $\mathbf{S}^{2}$. To account for this peculiarity [4], notation is changed to $\widetilde{W}_{\mu}^{\left(\frac{1}{2} \frac{1}{2}\right)}$. As an immediate application, the corresponding $\widetilde{W}^{2}$ in the product space, be it in $\psi_{\mu}$, or, $\Psi$, splits these spaces into covariant sectors corresponding to different $\widetilde{W}^{2}$ eigenvalues according to

$$
\left(\widetilde{W}^{\left(\frac{1}{2} \frac{1}{2}\right)} \otimes 1_{4}+1_{4} \otimes W^{\left(\frac{1}{2}, 0\right) \oplus\left(0, \frac{1}{2}\right)}\right)^{2} \Psi^{\tau_{l}}=-\tau_{l} m^{2} \Psi^{\tau_{l}}, \tau_{l}=\left(l \pm \frac{1}{2}\right)\left(l \pm \frac{1}{2}+1\right) .(6)
$$

Because of $\left[\widetilde{W}^{2}, \mathbf{S}^{2}\right] \neq 0$, the $\tau_{l}$ sectors (with $l=1,0$ ) describe boosted "has-been" spin$\left(l \pm \frac{1}{2}\right)$ states in the rest frame and states of undetermined spin in flight. On mass shell, $p^{2}=m^{2}$, Eq. (6) can be cast into the form (compare Ref. [3])

$$
P^{\frac{15}{4}}(\mathbf{p}) \Psi^{\frac{15}{4}}(\mathbf{p})=\Psi^{\frac{15}{4}}(\mathbf{p}), \quad P^{\frac{15}{4}}(\mathbf{p})=-\frac{1}{3}\left[\frac{1}{m^{2}} \widetilde{W}^{2}+\frac{3}{4}\left(1_{4} \otimes 1_{4}\right)\right] .
$$

It is easily verified that the operators $P^{\frac{15}{4}}(\mathbf{p})$, and $P^{\frac{3}{4}}(\mathbf{p})=1_{16}-P^{\frac{15}{4}}(\mathbf{p})$ are covariant projectors onto the $\widetilde{W}^{2}$ invariant subspaces with $\tau_{1}=\frac{15}{4}$, which is a parity singlet, and the two states of opposite parities with $\tau_{1}=\tau_{0}=\frac{3}{4}$, respectively, i.e.

$$
\left[P^{\frac{15}{4}}(\mathbf{p})\right]^{2}=P^{\frac{15}{4}}(\mathbf{p}), \quad\left[P^{\frac{3}{4}}(\mathbf{p})\right]^{2}=P^{\frac{3}{4}}(\mathbf{p}), \quad P^{\frac{15}{4}}(\mathbf{p}) P^{\frac{3}{4}}(\mathbf{p})=0 .
$$


In Ref. [5] we showed that the second auxiliary condition in the Rarita-Schwinger framework, $\gamma^{\mu} \psi_{\mu}=0$, is a short-hand from Eq. (7) and is determined as

$$
\frac{1}{m \alpha_{l}} \gamma^{\varepsilon}\left(\widetilde{W}_{\varepsilon \eta}^{\left(\frac{1}{2}, \frac{1}{2}\right)} \cdot \gamma \gamma_{5}\right)\left(\psi^{\tau_{l}}\right)^{\eta}(\mathbf{p})=\gamma \cdot \psi^{\tau_{l}}(\mathbf{p}) .
$$

Here, $\alpha_{l}$ is the eigenvalue of $\left[\widetilde{W}^{\left(\frac{1}{2} \frac{1}{2}\right)} \otimes 1_{4}\right] \cdot\left[1_{4} \otimes W^{\left(\frac{1}{2}, 0\right) \oplus\left(0, \frac{1}{2}\right)}\right]$ with respect to $\psi_{\mu}^{\tau_{1}}$. Upon using Eq. (2), and $p^{2}=m^{2}$, Eq. (8) can be cast into the form

$$
P^{\frac{15}{4}}(\mathbf{p})=-\frac{1}{3}\left[-\frac{1}{2} S \cdot S+\frac{1}{m^{2}} G \cdot G+\frac{3}{4}\left(1_{4} \otimes 1_{4}\right)\right] .
$$

Propagators and Lagrangians for $\widetilde{W}^{2}$ invariant sub-spaces. In having favored the 16 dimensional vector column space $\Psi$ over $\psi_{\mu}$, we gained the advantage that the Dirac equation has been automatically accounted for by means of the definition of the Lorentz generators within $\Psi$, i.e. through the second term on the rhs in Eq. (5). The propagator associated with Eqs. (7), (10) is now deduced as the following $16 \times 16$ matrix:

$$
\mathscr{S}^{\frac{15}{4}}(\mathbf{p})=\frac{\widetilde{P}^{\frac{15}{4}}(\mathbf{p})}{p^{2}-m^{2}}=\frac{2 S \cdot S-\frac{4}{m^{2}} G \cdot G-3\left(1_{4} \otimes 1_{4}\right)}{12\left(p^{2}-m^{2}\right)} .
$$

It directly verifies that this propagator corresponds to the following Lagrangian

$$
\mathscr{L}^{\frac{15}{4}}=\bar{\Psi}^{\frac{15}{4}}\left[2 m^{2} S^{\mu v} S_{\mu v}-4 S_{\mu v} p^{\mu} S^{\rho v} p_{\rho}\right] \Psi^{\frac{15}{4}}-15 m^{2}\left(1_{4} \otimes 1_{4}\right) \bar{\Psi}^{\frac{15}{4}} \Psi^{\frac{15}{4}} .
$$

Energy-momentum dispersion relations in the presence of electromagnetic fields. We here studied the energy-momentum dispersion relation of Eq. (7) in the presence of a simple magnetic field oriented along the $z$ axis, here denoted by $B_{z}$. We also took for the sake of simplicity of the calculation the $z$ axis along $\mathbf{p}$. With the help of the symbolic code Mathematica we then calculated the appropriate determinant and, in nullifying it, found the energy-momentum dispersion relation to be

$$
E^{2}=\left(p_{z}-e B_{z}\right)^{2}+m^{2}
$$

and therefore free of the Velo-Zwanziger problem of complex energy in the background of a magnetic field. Therefore, the associated interacting propagators can now be obtained in the standard way by replacing $\not p$ through $\hbar:=\left(p^{\mu}-e A^{\mu}\right) \gamma_{\mu}$. In having done so, we have produced a pathology-free propagating $\tau_{1}=\frac{15}{4}$ sector in the presence of an electromagnetic field. Admittedly, we did not produce pure-spin propagators. Nonetheless, we created a formalism that at least allows for the covariant description of the propagating "has-been" spin- $\frac{3}{2}^{-}$piece of the polar vector spinor. 


\section{REFERENCES}

1. G. Velo and D. Zwanziger, Phys. Rev. 186, 1337-1342 (1969).

2. L. H. Ryder, Quantum field theory (Cambridge Univ. Press, Cambridge,1987).

3. M. Kirchbach, Mod. Phys. Lett. A12, 3177-3188 (1997).

4. M. Kirchbach and D. V. Ahluwalia, Phys. Lett. B529, 124-131 (2002).

5. M. Kirchbach and D. V. Ahluwalia, hep-ph/0210084. 